(iii)轉化染的影响: 任反应过程中, 随着轉化來

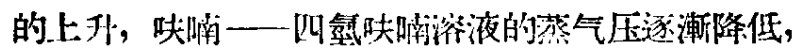

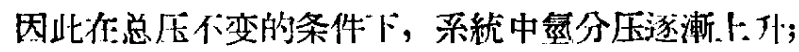
但是乍：一般情况下，其效应不甚显著; 唯作高溫低压 条作下, 其影响至为明显, 反应速度随着轉化率卜外 而上外(实驗 60 )。

5. 后期反应速度：当轉化率 超 过 $50 \%$ 以后, 反 应速度逐漸开始下降，四为四氢呋喃的洤度增高到一 定值后, 其解吸过程对於整个反应而言, 在动力学上. 有了显著的意义。泪度和压力对於后期反应速度的影 响你在下列規律:

(i) 反应速度开始下降的起点, 随埥所用压力的 增高而逐漸向高轉化率的方向推进; (ii)随着反应溫 度的提高, 反应速率随轉化率的变化率迅速減小。

所以提高压力和溫度, 对於后期反应速率有显著 的利晙，尤其是溫度的效应更为明显。

6. 如採朋 $190^{\circ} \mathrm{C}, 105$ 大气压, 加氫反应可以在 13分䤵內完成(实驗 58), 这为以流动系統进行高

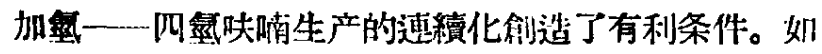
要提高反应速度, 压力虽可以进一步提高, 但是为了 避免副产率的上利, 溫度不应超过 $160-170^{\circ} \mathrm{C}$ 。

在探用热压釜进行分批式的生产时, 如要求使用 䡆低光压力, 作者据动力学数据进行了計算, 認为 30 大气压, $160^{\circ} \mathrm{C}$ 是最适宜的反应条件 (实驗 60), 反应可在 $100-120^{\circ}$ ( ) 危右开始, 利用反应热自动外 淄到 $160^{\circ} \mathrm{C}$ 。但在实际生产上, 似应探用 $160^{\circ} \mathrm{C}$ 和

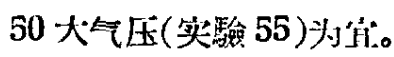

7.呋暔加妞如璪作不慎, 会有爆炸現像發生。高 压釜中的內容物应經常地保持在有效的撜䢁或振湯狀 态下, 避免局部过热和氫气流突然的冲沶, 这是防归:

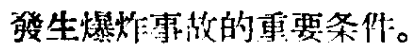

$$
\text { 汪仁严之光 李世亚 }
$$

(华东化工学院, 中国科学院化学研然所) 1058 年 7 月 8 日

\section{用格林函数解实际地形边界下 的斜压大气数值預报問題.}

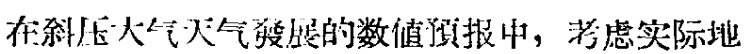
形上的运动学边照条件已有了方案和倾向計算的藏

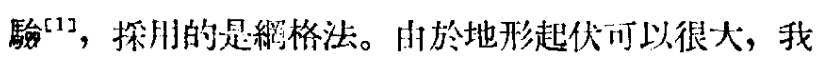

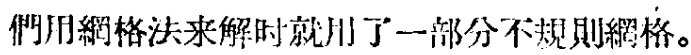

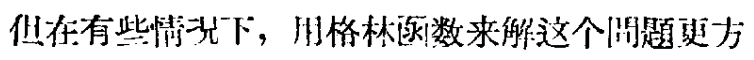

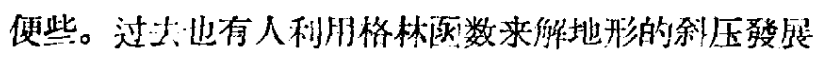

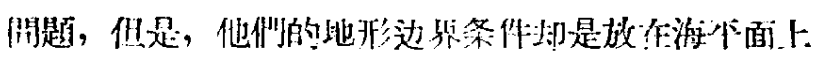

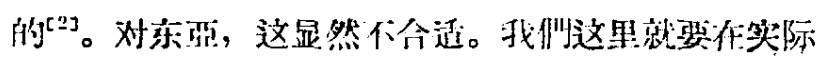
边柴条件下来解这个閵題。
把渦度方程写成

$$
\frac{\partial^{2} q}{\partial x^{2}}+\frac{\partial^{2} q}{\partial y^{2}}+\frac{\partial}{\partial \zeta}\left(\zeta^{2} \frac{\partial q}{\partial \zeta}\right)=F
$$

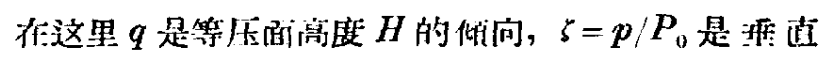
坐标, $P_{0}$ 是渡准面标准气医。 $F=-\left(c^{2} / l g\right) A_{Q}+$ $(R / g) \partial\left(\zeta A_{T}\right) / \partial \zeta 。 A_{0}, A_{T}$ 冬代袁渦度和溫度的华流。 $c^{2}=\alpha R T, \alpha=R / g\left(\gamma_{a}-\gamma^{\prime}\right) 。 l=2 \omega \sin \varphi$ 是地轉育数, 都 是已知的。它的边愁条件是

$$
\begin{gathered}
\zeta \rightarrow 0 \text { 时 } \zeta \frac{\partial q}{\partial \zeta} \text { 有界 } \\
\zeta=\zeta_{0}(x, y) \text { 时 } \quad\left(\zeta^{2} \frac{\partial q}{\partial \zeta}+x q\right)_{\zeta=\zeta_{0}}=\zeta \frac{k}{g}\left(A_{T}\right)_{\zeta=\zeta_{0}} \text { 一 } \\
-\frac{c^{2}}{l}(H, \eta)_{l=\zeta_{0}}
\end{gathered}
$$

这里 $\eta$ 是地形高度。( $A, B)$ 是信面 Jacobian，其他㹥 号如动力气象里一般得法。由於有地形起伏 $\zeta_{11} \neq 1$ 。

現在我們用 Вyлeeв 和 Марчук ${ }^{[2]}$ 的方式来解这 个方秙得到

$$
\begin{gathered}
q\left(x, y, \zeta^{\zeta}\right)=\frac{c^{2}}{2 \pi g} \int_{0}^{\zeta_{0}} \iint_{-\infty}^{+\infty} G_{L^{\prime}}^{H^{*}} A \Omega d x_{1}^{\prime} d y_{1}^{\prime} d \zeta^{\prime}- \\
-\frac{R}{2 \pi g} \int_{0}^{\zeta_{0}} \int_{-\infty}^{+\infty} \int_{-\infty}^{-} G_{T^{\prime}}^{H_{i}} A_{i} d x_{1}^{\prime} d y_{1}^{\prime} d \zeta^{\prime}-\frac{c^{2}}{2 \pi l} \iint_{-\infty}^{+\infty} \frac{1}{\sqrt{\zeta \zeta_{0}}} X_{\alpha} \\
\left(\ln \frac{\zeta_{0}}{\zeta}, r_{1}\right)(H, \eta)_{\zeta=\zeta_{0}} d x_{1}^{\prime} d y^{\prime}
\end{gathered}
$$

这里格林酷数

$$
\begin{aligned}
& G_{o}^{H_{s}}=\frac{1}{2 \sqrt{\zeta \zeta}}\left[\sigma\left(\left|\ln \zeta / \zeta^{\prime}\right|, r_{1}\right)-\sigma\left(\left|\ln \zeta_{0}^{2} / 5 \zeta\right|, r_{1}\right)\right. \\
& +2 X_{\alpha}\left(\ln \zeta_{0}^{2} / \zeta \zeta^{\prime}, r_{1}\right) \\
& G_{T}^{H_{t}}=\frac{1}{2 \sqrt{\zeta \zeta^{\prime}}}\left[\frac{1}{2} \sigma\left(\left|\ln \zeta^{\prime} / \zeta\right|, r_{1}\right)-\frac{1}{2} \sigma\left(\mid \ln \zeta_{0}^{\prime} / \zeta \zeta^{\prime}, r_{1}\right)\right. \\
& \left.+2 X_{\alpha}\left(\ln \zeta_{0}^{2} / \zeta \zeta^{\prime}, r_{1}\right)-\hat{\imath} \sigma \mid \begin{array}{l|l}
\hat{\imath} \omega & a=\left|\ln \zeta^{\prime} / \zeta\right| \\
& a=\ln \zeta_{0}^{2} / \zeta \zeta
\end{array}\right] \\
& \sigma\left(a, r_{1}\right)=\int_{0}^{\infty} \frac{1}{\mu} e^{-\mu a} J_{n}\left(\rho, r_{1}\right) \rho d \rho \\
& X_{a}\left(a, r_{1}\right)=\int_{0}^{\infty} \frac{e^{-\mu a}}{\mu-\frac{1}{2}+\alpha / \zeta_{0}} J_{0}\left(\rho, r_{1}\right) \rho d \rho
\end{aligned}
$$

注意这里更直方问积分与限是 $\zeta_{11}=\zeta_{11}(x, y)$, 水水

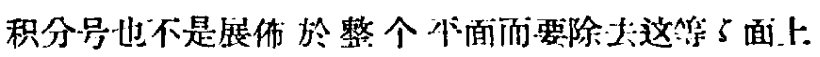
地形所佔去的那一塊。还要注:意作格林河数里全有 $\zeta_{0}(x, y)$ 。刚此, 对於不问点, 的 $q$, 格林源数的值逐

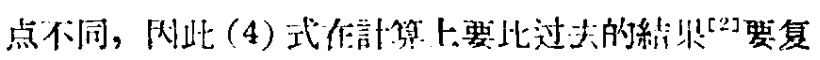
杂得多。

$$
\text { 王 宗 晧 }
$$

( 中国科学院地球物理呼哞所)

1 月58 6 月7日 


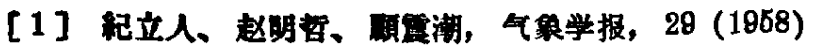
(排印中)。

[2] Кибель, И. А., Введение в гидродинамитескпе методы краткосропного прогноза погоды, 1957 , Mосква.

\section{目洞的一种可能成因}

关於鼠洞的成因, 多数学者解釋为末被溶液全部 充㙋, 或因后期溶液溶解而重結疁所致 ${ }^{[1]}$ 。但这些解 瀷与作者在野外所覌察啲的晶洞份有出入。現就作者 在江:西含暏不英脈所进行的覌察提出以下㻎法:

(一)晶洞分佈的特点: 本区昆洞常分作在脈的中 部, 成透鏡呺, 長輣多与走向坐行, 有时長可达20厘 米。其中大多仅有石英晶但有时为黄鉄矿及其他 硫化物充埧。'烝洞較大时, 其底部被大量板狀及片彇 石英晶体充塂。总的說来，分佈在脈中的毘润的分作 規律是: 在上部中段或胍的尖端及尖灭处䐯洞較多, 而且一般較小夕眼下部疁洞少而大。

但昆润也可在胍的边部你在, 而且详非个别現 象, 此时晶洞的延長方向也与腿的走向平行。零都瞥

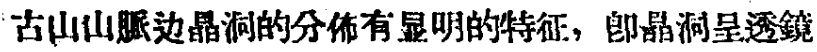

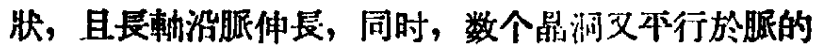
走向連成一电, 部分昆洞則互相連接做若本行於胍的 裂維。昆洞网壁均有晶头很短的石英晶体（圆 1)。

佾有少量晶洞的分佈与上迌特点不一读, 即完全 蛋近㭽壁，是不規則形㸝。

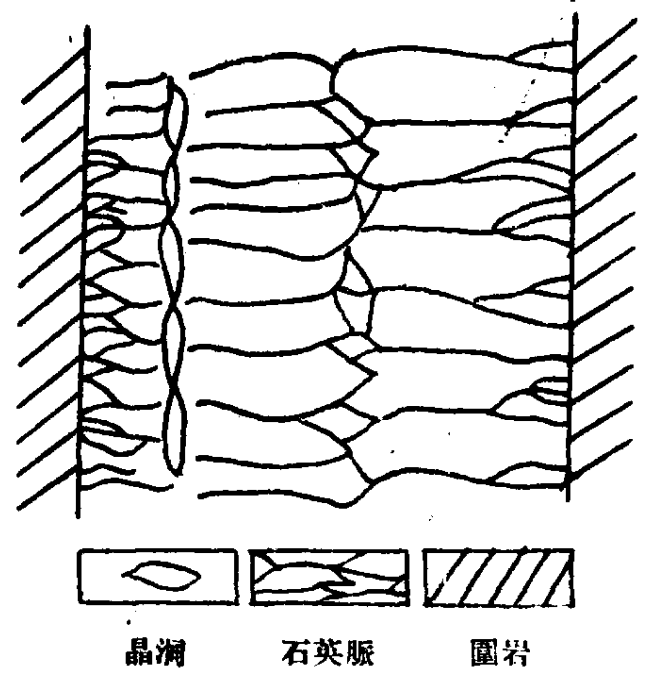

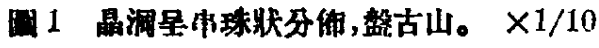

（二.)毘润形成的可能原因：过去对於昆洞形成的 原因, 一般認为, 上一个济液局部被生長石英所阴，因

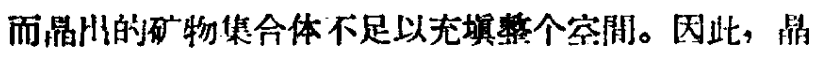
洞多惟現在脤的中部, 且沿脈的走向延長, 这可解釋 矿眼上部及矿展变寉，尖灭处昆润加多的現象，因

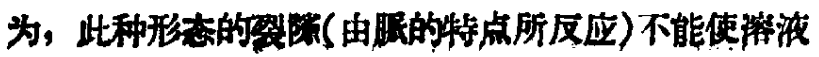

的供給完全淂通無阴。

經过野外实地覌查, 作者認为.上述形成晶洞的一

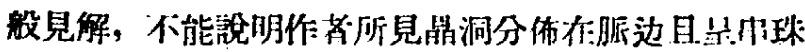
狀的現象，因此提出，裂㭞重复張开及局部扩大是形 成晶洞，特别是后一种喿洞的重要原因。由於裂隐的 里复張开, 不但可引起再次或多次的充填, 另外也可 使生長石英昆体的脆弱部分墢生裂隐。目於后期溶液

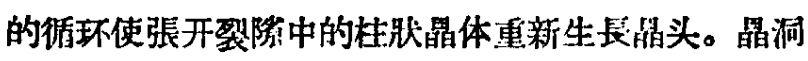
底部的板快及片状石英鮊体是裂隐張开时掉落的碎片 再生的結果。这样，出現在石英脈边部的电珠狀昆洞 的形态及分作特点就很自然的了。脈中部分毘洞的形 成也可用这种原因来解釋，如鉄川1壠胃有一条脈中部

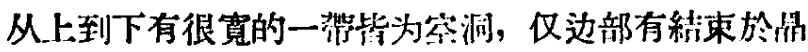
渼阶段的毘体充宪。

（三)裂除重复張开的現像的实例：这种現象几乎

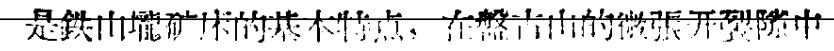

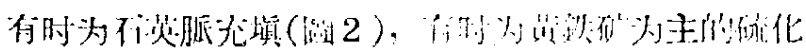

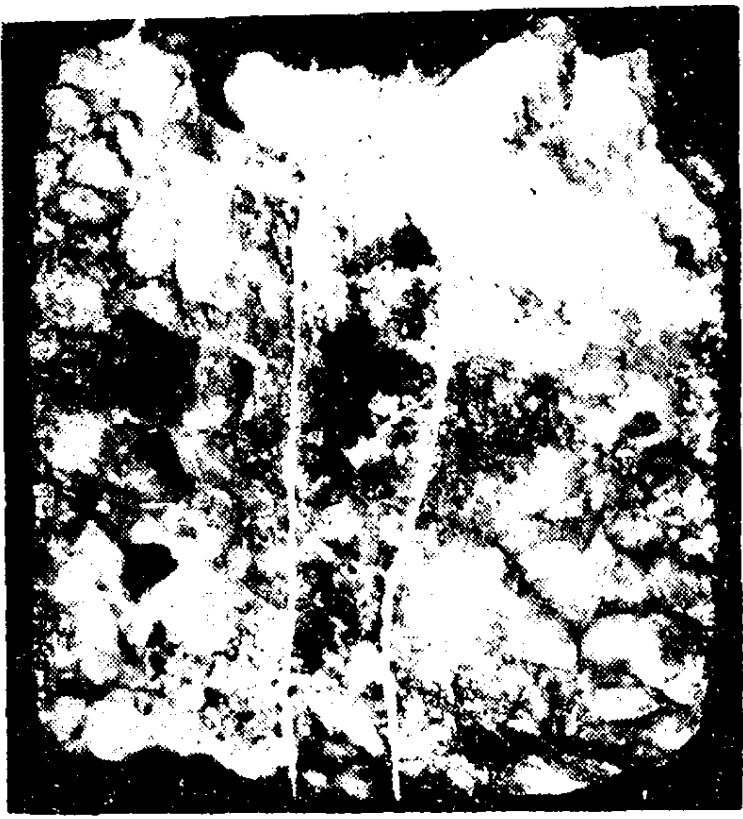

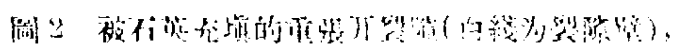
瞥古比。 $\times 2 / 3$

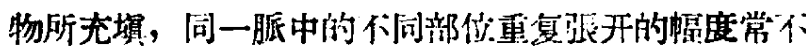

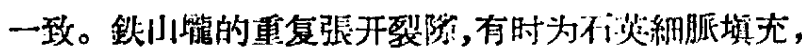

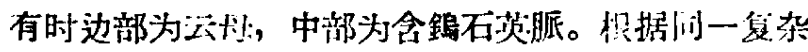

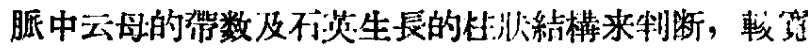
的复杂矿脈常是 $3-5$ 次重張开裂陆坞态嫔的絬果。

朱 而勤

(唇春地貿斯堔学院)

1958 年 6 If 24 日

[1] A. E. 卡格: 水晶洞的形成过积, 地知識, 1955,10 期。

[2] Д. В. Рундквист, Вап. Весе, Минер, пй-ва. 1957, Bum. B, 